\title{
ADAMTS Sol narae cleaves extracellular Wingless to generate a novel active form that regulates cell proliferation in Drosophila
}

\author{
Jong-Hoon Won ${ }^{1}$, Go-Woon Kim¹, Ja-Young Kim, Dong-Gyu Cho' ${ }^{1}$ Buki Kwon', Young-Kyung Bae $\mathbb{E}^{2}$ and \\ Kyung-Ok Cho ${ }^{1}$
}

\begin{abstract}
s
Wnt/ Wingless $(\mathrm{Wg})$ is essential for embryonic development and adult homeostasis in all metazoans, but the mechanisms by which secreted Wnt/Wg is processed remain largely unknown. A Drosophila Sol narae (Sona) is a member of $\mathbf{A}$ Disintegrin $\mathbf{A}$ nd $\mathbf{M e t a l l o p r o t e a s e}$ with ThromboSpondin motif (ADAMTS) family, and positively regulates Wg signaling by promoting Wg secretion. Here we report that Sona and Wg are secreted by both conventional Golgi and exosomal transports, and Sona cleaves extracellular $\mathrm{Wg}$ at the two specific sites, leading to the generation of $\mathrm{N}$ terminal domain (NTD) and C-terminal domain (CTD) fragments. The cleaved forms of extracellular Wg were detected in the extracellular region of fly wing discs, and its level was substantially reduced in sona mutants. Transient overexpression of Wg-CTD increased wing size while prolonged overexpression caused lethality and developmental defects. In contrast, Wg-NTD did not induce any phenotype. Moreover, the wing defects and lethality induced by sona RNAi were considerably rescued by $\mathrm{Wg}-\mathrm{CTD}$, indicating that a main function of extracellular Sona is the generation of Wg-CTD. Wg-CTD stabilized cytoplasmic Armadillo (Arm) and had genetic interactions with components of canonical Wg signaling. Wg-CTD also induced Wg downstream targets such as Distal-less (DII) and Vestigial (Vg). Most importantly, Cyclin D (Cyc D) was induced by Wg-CTD but not by full-length Wg. Because Sona also induces Cyc D in a cell non-autonomous manner, Wg-CTD generated by Sona in the extracellular region activates a subset of Wg signaling whose major function is the regulation of cell proliferation.
\end{abstract}

\section{Introduction}

Cellular communication via components in the extracellular matrix (ECM) is essential for cell survival and proliferation as well as differentiation. Extracellular proteases play important roles in regulating activity, localization and stability of the ECM proteins ${ }^{1-3}$. Despite the importance of these proteases, their specific functions are

Correspondence: Kyung-Ok Cho (kocho@kaist.ac.kr)

'Department of Biological Sciences, Korea Advanced Institute of Science and Technology, 291 Daehak-ro, Yuseong-gu, Daejeon, Korea

${ }^{2}$ Center for Bioanalysis, Korea Research Institute of Standards and Science, 267 Gajung-ro, Yuseung-gu, Daejeon, Korea

Edited by E. Baehrecke still largely unexplored. ADAMTS family contains extracellular proteases that are present only in metazoans ${ }^{4,5}$. Six and nineteen members have so far been identified in flies and mammals, respectively ${ }^{6,7}$. Mammalian ADAMTSs are involved in cell proliferation, angiogenesis and organogenesis, so their malfunctions result in various diseases such as cancer, arthritis, and arteriosclerosis ${ }^{7-9}$. An ADAMTS Sol narae (Sona) is essential for fly development ${ }^{10}$. Loss of sona decreases the level of extracellular $\mathrm{Wg}$, and sona exhibits positive genetic interaction with wntless (wls) that encodes a cargo protein for $\mathrm{Wg}^{10-12}$. Therefore, intracellular Sona seems to cooperate with Wls in Wg secretion ${ }^{10}$. We recently reported a new function of

\section{(c) The Author(s) 2019}

(c) (i) Open Access This article is licensed under a Creative Commons Attribution 4.0 International License, which permits use, sharing, adaptation, distribution and reproduction c. in any medium or format, as long as you give appropriate credit to the original author(s) and the source, provide a link to the Creative Commons license, and indicate if changes were made. The images or other third party material in this article are included in the article's Creative Commons license, unless indicated otherwise in a credit line to the material. If material is not included in the article's Creative Commons license and your intended use is not permitted by statutory regulation or exceeds the permitted use, you will need to obtain permission directly from the copyright holder. To view a copy of this license, visit http://creativecommons.org/licenses/by/4.0/. 
extracellular Sona in cell survival and cell proliferation ${ }^{13}$. sona has genetic interactions with cell death-related genes such as Death-associated inhibitor of apoptosis (Diap1) and reaper. Interestingly, Sona upregulates Cyclin D (Cyc D) in a cell non-autonomous manner, and increases tissue size. Cyc D is a G1 Cyclin to initiate the cell cycle by responding to the mitogen signals ${ }^{14}$. Therefore, it is possible that extracellular Sona generates a yet unidentified signaling molecule that induces Cyc D in the signal-receiving cells.

Wnt family is essential for animal development, and has been extensively studied since a mutant of fly Wg, the homolog of vertebrate Wnt1, was described a century ago $^{15-17}$. Wnt is secreted by both conventional Golgimediated transport and exosomal secretion pathway ${ }^{18-20}$. Interaction between Wnt and Frizzled (Fz) receptors initiates a cascade of intracellular responses in the responding cells that lead to downstream gene expression ${ }^{21,22}$. In flies, $\mathrm{Wg}$ is involved in cell proliferation, differentiation, and survival by inducing Wg effector components including Vestigial (Vg), Distal-less (Dll) and Senseless (Sens) ${ }^{23-26}$. In mammals, Wnt signaling promotes cell proliferation by transcriptional activation of multiple target genes such as cMyc and Cyc $\mathrm{D}^{27-29}$ and its malfunction leads to various diseases such as cancer, neurodegenerative diseases, inflammatory disease, and diabetes ${ }^{30-32}$.

We asked the role of extracellular Sona in this study and found that Sona generates NTD and CTD fragments of Wg by cleaving extracellular Wg. The Wg-CTD fragment was similar to full-length $\mathrm{Wg}$ in activating canonical $\mathrm{Wg}$ signaling but was dissimilar to full-length Wg in Cyc D induction, lack of Sens induction, and protein instability. Thus, one of the main functions of Sona is to generate Wg-CTD that carries out subsets of Wg signaling.

\section{Results}

Sona and $\mathrm{Wg}$ are secreted by both conventional Golgi and exosomal transports

Sona is an ADAMTS protease secreted as an active form to the extracellular region ${ }^{10}$, and has biochemical and genetic interactions with $\mathrm{Wg}$ that is secreted by both conventional Golgi and exosomal transports ${ }^{18-20}$. As a first step toward understanding the role of extracellular Sona, we examined by which pathway Sona is secreted. To this end, we obtained conditioned media from the culture of $S 2$ sona-HA cell line, and precleared it to obtain the initial supernatant fraction, $\mathrm{SN}_{0}$. Centrifugation of $\mathrm{SN}_{0}$ at $100,000 \times g$ yielded two fractions: a supernatant fraction $\left(\mathrm{SN}_{\Delta}\right)$ that contains soluble proteins secreted by Golgi transport, and a pellet fraction (P100) that contains exosomes $^{19,33}$. The cell extract (CX) contained both fulllength Sona-HA (red arrow) and the active form of Sona$\mathrm{HA}$ lacking its pro-domain (black arrow), while the $\mathrm{SN}_{\Delta}$ and P100 fractions contained only the active form of
Sona-HA (Fig. 1a). Our data demonstrate that Sona is also secreted by both Golgi and exosomal transports (Fig. 1b). To prove further that Sona is secreted by exosomal pathway, we examined the P100 fraction of Sona-HA in detail. Purity of the P100 fraction was verified by the presence of the exosomal markers Syntaxin 1A (Syx1A) and Alix as well as the absence of the ER marker Calnexin $^{33,34}$ (Fig. 1c). Particulate structures with 70-250 nm diameter were detected in the P100 fraction of S2 sona$H A$ or S2 GFP-wg by Nanoparticle tracking analysis (NTA), and Sona and Wg were present in the fraction with $1.09-1.11 \mathrm{~g} / \mathrm{mL}$ density in sucrose step gradient ${ }^{33}$ (Fig. 1d and Supplemental Fig. S1A-B). Furthermore, Sona-HA was also present on the outer surface of vesicles (Fig. 1e and Supplemental Fig. S1C, D). To confirm the presence of Sona on exosomes in vivo, we examined whether Sona-HA and the exosomal marker CD63-GFP colocalize in wing discs. Unlike the lysosomal markers (Supplemental Fig. S2), half of CD63-GFP-positive $(+)$ vesicles contained Sona-HA detected by both anti-HA and Sona-Pro antibodies $(52.3 \pm 10.3 \%, n=65$, Supplemental Fig. S1E) ${ }^{10}$, and about half of these CD63+ Sona+ vesicles contained endogenous $\mathrm{Wg}(21.5 \pm 2.1 \%, n=65$, Supplemental Fig. S1F). These results demonstrate that Sona is present on exosomes.

\section{The active form of extracellular Sona is essential for cleavage of the $\mathrm{Wg}$ linker region}

Coimmunoprecipitation of Sona and $\mathrm{Wg}^{10}$ suggested that Sona may cleave Wg. To test this, we examined whether any small $\mathrm{Wg}$ fragments are generated in the presence of Sona. In fact, a $65 \mathrm{kDa}$ fragment smaller than $83 \mathrm{kDa}$ full-length GFP-Wg (GFP-Wg ${ }^{\mathrm{FL}}$ ) was detected by the 4D4 Wg antibody in both $\mathrm{SN}_{\Delta}$ and $\mathrm{P} 100$ fractions only when Sona was coexpressed with GFP-Wg (black arrows in Fig. 1f', f'). Such Sona-dependent changes were not detected in CX (Fig. If and Supplemental Fig. S3A, B). Interestingly, anti-GFP antibody detected both $65 \mathrm{kDa}$ and an additional $60 \mathrm{kDa}$ fragment (black arrowhead in Fig. 1h), indicating that these two fragments have the Nterminal region where GFP is inserted (Fig. 1k). Since these 65 and $60 \mathrm{kDa}$ fragments were not produced by the protease-dead SonaE475A or in the presence of a zinc chelator, EDTA, metalloprotease activity of Sona is essential for Wg cleavage (Fig. 1g, h and Supplemental Fig. S3A-C). We located the two cleavage sites designated as L1 and L2 in the Wg linker based on two features. First, the $65 \mathrm{kDa}$ but not the $60 \mathrm{kDa}$ fragment has the 4D4 epitope (Fig. 1g, h). Second, the 4D4 epitope is located between amino acids 229 and 360 of $\mathrm{Wg}^{35,36}$. Cleavage at L1 and L2 should produce fragments named NTD ${ }^{\mathrm{L} 1}$, $\mathrm{CTD}^{\mathrm{L} 1}$, NTD ${ }^{\mathrm{L} 2}$, and $\mathrm{CTD}^{\mathrm{L} 2}$ (Fig. 1k). NTD ${ }^{\mathrm{L} 1}$ and NTD ${ }^{\mathrm{L} 2}$ are $60 \mathrm{kDa}$ and $65 \mathrm{kDa}$ fragments, respectively. Meanwhile, a $23 \mathrm{kDa}$ fragment was detected with the $4 \mathrm{D} 4$ 


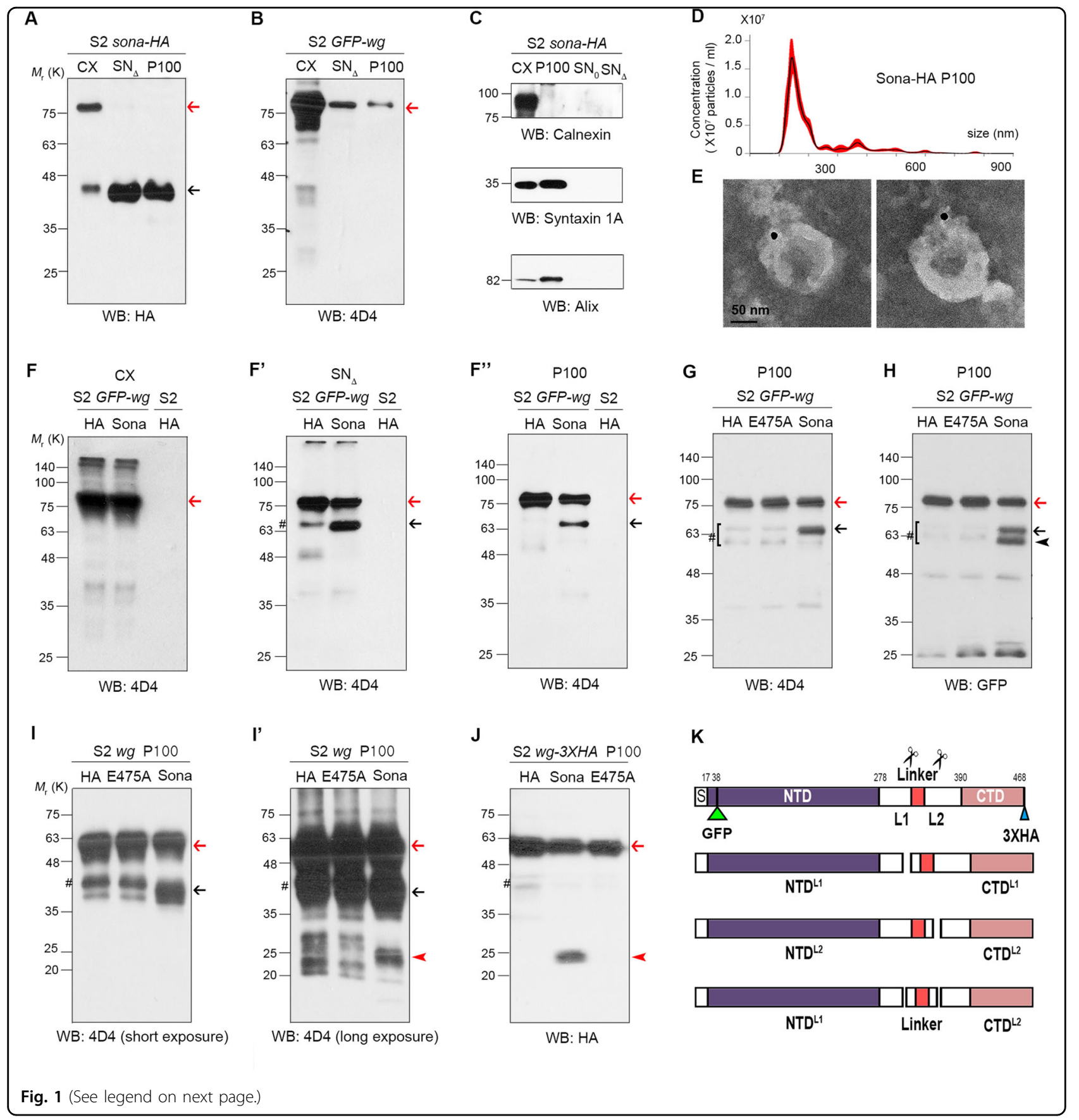

antibody when untagged Wg or GFP-Wg was coexpressed with Sona (red arrowheads in Fig. 1i' and Supplemental Fig. S3A'), or detected with the HA antibody when Sona and Wg-3XHA with three HA tags at the C-terminus ${ }^{37}$ were co-expressed (red arrowhead in Fig. 1j). Therefore, this $23 \mathrm{kDa}$ fragment is $\mathrm{CTD}^{\mathrm{L} 1}$ because it contains both the C-terminal part of $\mathrm{Wg}$ and the 4D4 epitope (Fig. 1k). However, $C^{2} D^{\mathrm{L} 1}$ fragments generated from the untagged Wg or GFP-Wg were detectable only after long exposure, and the intensity of $\mathrm{CTD}^{\mathrm{L} 1}$ fragment was much lower than that of $\mathrm{NTD}^{\mathrm{L} 2}$ (Fig. 1i-i' and Supplemental Fig. S2A'). Moreover, the $\mathrm{CTD}^{\mathrm{L} 2}$ fragment was never detected when Wg-3XHA was coexpressed with Sona (Fig. 1j). The low levels of $\mathrm{CTD}^{\mathrm{L} 1}$ and the absence of the CTD ${ }^{\mathrm{L} 2}$ may be due to protein instability, which will be addressed in Fig. 2.

\section{The linker of $\mathbf{W g}$ is necessary and sufficient for $\mathbf{W g}$ cleavage by Sona}

To examine whether the linker is the only region required for Wg cleavage, we generated two constructs, 
(see figure on previous page)

Fig. 1 Active Sona is responsible for cleaving extracellular $\mathbf{W g}$ in its linker region. In all western analyses, blotting antibodies are indicated at the bottom of each panel. Fractions and the source of cells transfected with different constructs are written at the top of panels. S2 cells are derived from a macrophage-like lineage of fly embryonic cells ${ }^{78}$, and express neither Sona nor $\mathrm{Wg}^{10,19,33}$. Therefore, UAST-GFP-Wg and UAST-SOna-HA CDNA constructs were expressed by actin-Gal4 driver in S2 cells. HA is the preparation from cells transfected with the control UAST-HA vector. The pound signs (\#) indicate the cleaved Wg fragments in the absence of Sona. These fragments may be generated by degradation during sample preparation or by some Wg-specific proteases endogenously expressed in S2 cells. a Full-length and processed forms of Sona-HA in cell extract (CX), SN $\mathrm{N}_{\triangle}$, and P100 fractions. The P100 fraction was four times more concentrated than the $\mathrm{SN}_{\Delta}$ fraction, and the equal volumes of concentrated samples were loaded for the blot. Therefore, the actual amount of Sona in the $\mathrm{SN}_{\Delta}$ fraction should be four times more than the one in the blot. The $\mathrm{SN}_{\Delta}$ fraction always contained more Sona than the P100 fraction. Full-length Sona and active Sona are marked with red arrows and black arrow, respectively. b Full-length GFP-Wg (GFP-Wg ${ }^{\mathrm{FL}}$ ) in $\mathrm{CX}, \mathrm{SN}_{\Delta}$, and P100 fractions. $\mathrm{SN}_{\Delta}$ and P100 fractions were concentrated equally and the same volumes were loaded. GFP-Wg was more abundant in the $\mathrm{SN}_{\Delta}$ fraction than the P100 fraction (red arrow) ${ }^{19,33}$. c Verification of CX, P100, $S N_{0}$, and $S N_{\Delta}$ fractions using the ER marker Calnexin and the exosomal markers Alix and Syntaxin 1A (Syx1A). d Diameter and number of vesicles in P100 fractions from S2 sona-HA cells measured by NTA. e A immuno-EM image of Sona-HA in the P100 fraction with anti-HA antibody. The diameter of vesicles with Sona-HA is about 120 nm. f-f" GFP-Wg ${ }^{\mathrm{FL}}(83 \mathrm{kDa}$ ) and a GFP-Wg fragment (65 kDa) are indicated with red and black arrows, respectively. $\mathbf{g}$, $\mathbf{h}$ The black arrow and the arrowhead indicate 65 and $60 \mathrm{kDa}$ GFP-Wg fragments, respectively. The same blot was used for both (g) and (h). i A blot in (i) was exposed longer in (i'). The $41 \mathrm{kDa}$ and the $23 \mathrm{kDa}$ fragments are marked by the black arrows and the red arrowhead, respectively. Non-tagged $\mathrm{Wg}^{\mathrm{FL}}$ are marked by red arrows. $\mathbf{j}$ The $23 \mathrm{kDa}$ Wg-3XHA fragment are marked with the red arrowhead. This fragment was readily detected because it has three times HA epitope than 4D4 epitope. $\mathbf{k}$ Domain structures of full-length and cleaved forms of Wg (modified from a report ${ }^{79}$ ). Two cleavage sites $(\mathrm{L} 1$, L2) are indicated with scissors. The 4D4 epitopes are marked with red

GFP-NTD and linker-GFP that encode $53 \mathrm{kDa}$ GFP-NTD without the linker region and $48 \mathrm{kDa}$ Linker-GFP with the linker region fused to GFP, respectively (Fig. 2a, d). When GFP-NTD and Sona-HA were coexpressed, Sonadependent cleavage was not detected (Fig. 2c). In contrast, we found all cleavage products such as a $42 \mathrm{kDa} \mathrm{L1}$ cleavage product (black arrows), a $34 \mathrm{kDa}$ L2 cleavage product (red arrowheads), and a $12 \mathrm{kDa}$ L2 cleavage product (black arrowheads) when Linker-GFP and SonaHA were coexpressed (Fig. 2f, h). Furthermore, Wg cleavage by Sona occurred when Sona and Wg were prepared from different cells or different secretion pathways (Supplemental Fig. S3D-F). Therefore, the linker region is necessary and sufficient for Wg cleavage by Sona regardless of origin.

\section{The CTD domain of Wg-CTD fragments is responsible for protein instability}

We previously mentioned that both $\mathrm{CTD}^{\mathrm{L} 1}$ and $\mathrm{CTD}^{\mathrm{L} 2}$ fragments might be unstable (Fig. 1i). However, the $42 \mathrm{kDa}$ L1 Linker-GFP fragment that is equivalent to the $23 \mathrm{kDa}$ Wg-CTD ${ }^{\mathrm{L} 1}$ fragment was readily detected (Compare Fig. $1 \mathrm{i}$ to Fig. 2h). This raised a possibility that the CTD domain itself is responsible for instability of Wg-CTD fragments. To test this, we generated linker-CTD that encodes $29 \mathrm{kDa}$ Linker-CTD and compared its Sona-dependent cleavage products to those of Linker-GFP (Fig. 2i). When LinkerCTD and Sona were coexpressed, a $12 \mathrm{kDa}$ 4D4-positive L2 cleavage product was detected (black arrowheads) but the expected $23 \mathrm{kDa} \mathrm{CTD}^{\mathrm{L} 1}$ was not detected even with multiple attempts (Fig. 2k). Moreover, the $34 \mathrm{kDa}$ L2 linkerGFP fragment was detected (red arrowhead in Fig. 2f), which is equivalent to the $17 \mathrm{kDa} \mathrm{Wg-CTD}{ }^{\mathrm{L} 2}$ fragment. As shown before, this $17 \mathrm{kDa} \mathrm{Wg}^{-C T D}{ }^{\mathrm{L} 2}$ fragment was never detected throughout this study (Fig. 1j, k). Therefore, both
Wg-CTD fragments are less stable than their equivalents due to the CTD itself.

\section{Wg is directly cleaved by Sona}

To test the enzyme-substrate relationship between Sona and Wg, we asked whether Sona cleaves Wg in vitro. Since the NTD of Wg is too hydrophobic to be expressed in $E$. coli, we generated GST-linker-CTD (Fig. 2l). We hypothesized that Sona may cleave GST-linker-CTD because the linker is sufficient for cleavage by Sona (Fig. 2l). When GST-linker-CTD purified from $E$. coli was incubated with active Sona purified from the $\mathrm{SN}_{\Delta}$ fraction of S2 sona-HA (Supplemental Fig. S4A), the 4D4 antibody detected a Sona-dependent $18 \mathrm{kDa}$ fragment (red arrowhead in Fig. $2 \mathrm{~m}$ and Supplemental Fig. S4B; black arrow in Fig. 2n and Supplemental Fig. S4C). This $18 \mathrm{kDa}$ fragment is the L1 cleavage product that is equivalent to the $23 \mathrm{kDa} \mathrm{Wg}$ $\mathrm{CTD}^{\mathrm{L} 1}$ fragment, but is smaller because the CTD domain in the GST-linker-CTD is not glycosylated (Supplemental Fig. S4D). This suggests that Wg is a substrate of Sona.

If Sona directly cleaves $\mathrm{Wg}$, the amount of cleaved Wg would positively correlate with that of Sona. To test this, the fixed amount of $\mathrm{SN}_{0}$ containing $\mathrm{Wg}$ was incubated with the increasing amounts of $\mathrm{SN}_{0}$ containing Sona-HA. As expected, the amount of $\mathrm{Wg}-\mathrm{CTD}^{\mathrm{L} 1}$ fragment positively correlated with that of Sona (Fig. 20). When the fixed amount of the P100 containg GFP-Wg was incubated with the increasing amounts of the P100 containing Sona-HA, the amount of Wg-NTD ${ }^{\mathrm{L} 1}$ and $\mathrm{Wg}-\mathrm{NTD}^{\mathrm{L} 2}$ fragments also proportionally increased (Fig. 2p). This result is consistent with direct cleavage of Wg by Sona.

\section{Sona is required for cleavage of extracellular $\mathrm{Wg}$ in vivo}

We next asked whether cleavage of extracelluar Wg also occurs in vivo. $\mathrm{Wg}$ is highly expressed along the DV 


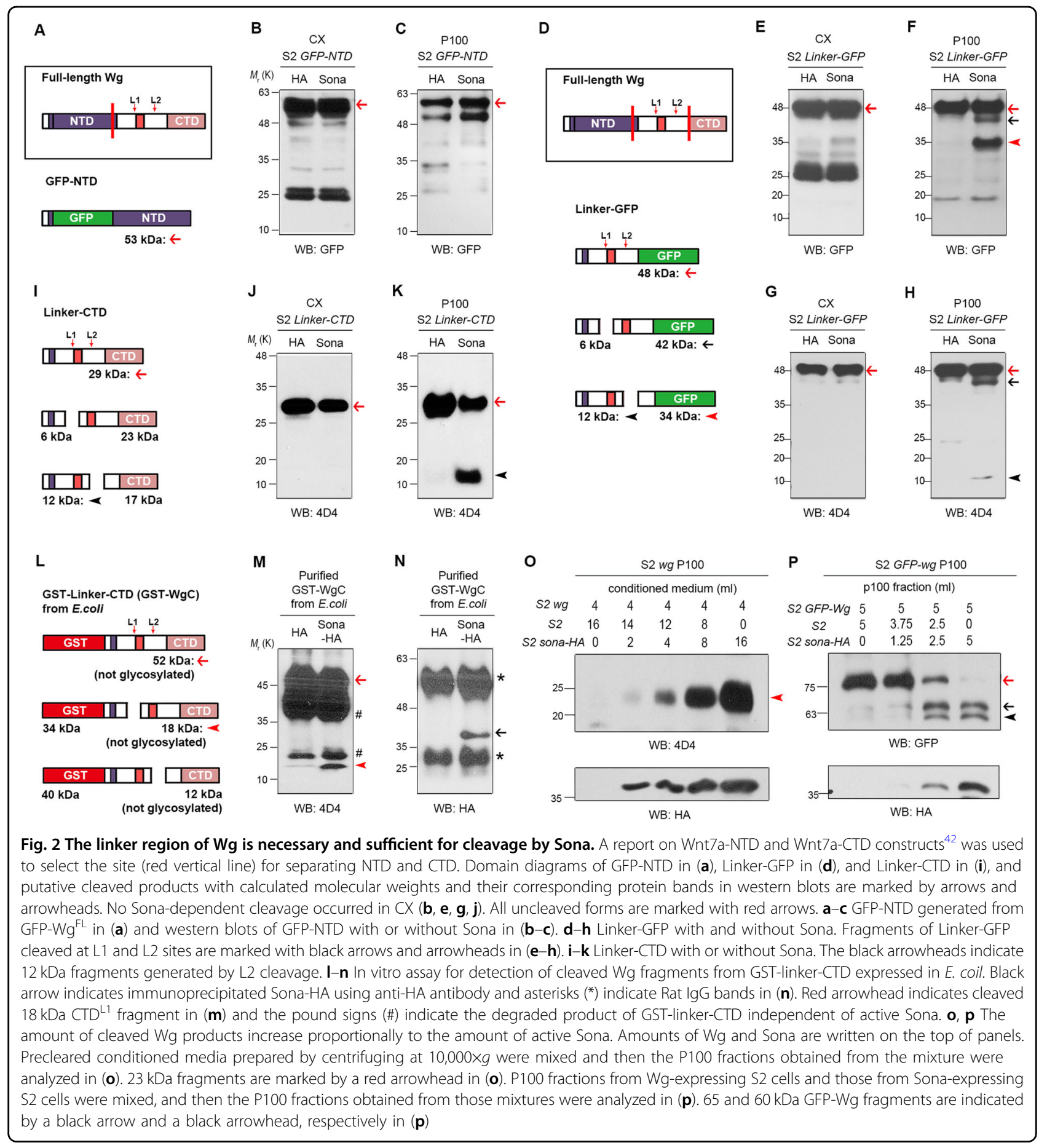

midline of wing discs ${ }^{38-40}$. To detect cleaved Wg forms, we examined extracellular Wg-HA pattern in the DV midline of $w g[\mathrm{KO} ; \mathrm{Wg}-H A]^{41}$. Assuming Sona cleaves $\mathrm{Wg}$ at L1 and L2 cleavage sites in vivo, we expected to detect extracellular structures including $\mathrm{Wg}-\mathrm{HA}{ }^{\mathrm{FL}}$ and four additional Wg fragments except $\mathrm{NTD}^{\mathrm{L} 1}$ in $w g[K O ; W g$ $H A]$ wing discs using anti-HA and 4D4 antibodies (Fig. 3a). We found not only yellow structures $\left(\mathrm{HA}^{+}\right.$
$4 \mathrm{D} 4^{+}$) that contain full-length Wg but also the green $\left(\mathrm{HA}^{+} 4 \mathrm{D} 4^{-}\right)$and the red structures $\left(\mathrm{HA}^{-} 4 \mathrm{D} 4^{+}\right)$that represent cleaved Wg fragments (Fig. 3a, e). These cleaved structures were also detected in $w g>G F P$-wg discs (Supplemental Fig. S5). Thus, cleaved Wg fragments were present in the extracellular region.

If Sona plays a major role in the cleavage of $\mathrm{Wg}^{\mathrm{FL}}$, sona mutants should have more $\mathrm{Wg}^{\mathrm{FL}}$ than wild-type. To test 
this, we compared the percentage of yellow structures $\left(\mathrm{HA}^{+} 4 \mathrm{D} 4^{+}\right)$in sona ${ }^{18} /$ sona ${ }^{47} w g[K O ; W g-H A]$ discs with that in control $w g[K O ; W g-H A]$ discs (Fig. 3d-h). While $74.1 \%(648 / 875)$ were yellow in sona ${ }^{18} /$ sona $^{47}$ discs, only $32.0 \%$ (460/1436) were yellow in wild-type discs (Fig. 3h). This suggests that Wg cleavage occurred at a lesser extent in the sona discs. This supports that Sona is a major player in cleavage of Wg in vivo.

\section{Prolonged overexpression of $\mathbf{W g}$-CTD induces morphological defects and lethality}

We reasoned that at least one of the cleaved forms of Wg should be active because Sona positively regulates Wg signaling ${ }^{10}$. To test which $\mathrm{Wg}$ fragment is active, we performed luciferase assay in $\mathrm{S} 2 \mathrm{R}+$ cells that expressed Wg-NTD or Wg-CTD. Neither Wg-NTD nor Wg-CTD, however, showed any Wg activity (Supplemental Fig. S6A). This was unexpected because artificially engineered Wnt7a-CTD is reported to be active in TopFlash reporter assay $^{42}$. It is possible that $\mathrm{S} 2 \mathrm{R}+$ cells lack some essential components that are required for Wg-CTD activity ${ }^{43}$, or Wg-CTD is too unstable in S2 R+ cell culture (Supplemental Fig. S6). Instead of finding the better condition for luciferase assay, we decided to test the activity of $\mathrm{Wg}$ fragments in vivo with $U A S-G F P$ - wg-NTD and $U A S$ - wg$m y c C T D$ transgenic flies (Fig. 2a and Supplemental Fig. S7A).

Overexpression of GFP-Wg-NTD with engrailed (en)Gal4 or nubbin (nub)-Gal4 produced no phenotypes (Fig. 4b and Supplemental Fig. S7B). In contrast, en $>w g$ $m y c C T D$ and $n u b>w g-m y c C T D$ wings were small and deformed (Fig. 4a, d, k, l), and the posterior region of en > $w g$-mycCTD wing discs were smaller than that of control discs (Supplemental Fig. S7K, L). Furthermore, cubitus interruptus $(c i)>w g-m y c C T D$ eyes were small and rough (Supplemental Fig. S7N, O). Expression of Wg-mycCTD by actin-Gal4 or tubulin-Gal4 induced early larval lethality $(n>40)$ but that of GFP-wg-NTD produced no lethality. Expression of the untagged Wg-NTD and WgCTD also generated phenotypes similar to the tagged counterparts (Fig. 4c, e and Supplemental Fig. S7A, E, F, J, $\mathrm{M})$. Taken together, Wg-CTD but not NTD is an active Wg form in vivo.

\section{Transient overexpression of Wg-CTD stimulates cell proliferation}

Wg stimulates cell proliferation as a mitogen ${ }^{44,45}$, and moderate $\mathrm{Wg}$ overexpression increases the number of phosphohistone 3 (PH3)-positive cells ${ }^{44,46}$. To check whether Wg-CTD also induces cell proliferation, we transiently expressed Wg-CTD using Gal80 ${ }^{t s}$ in order to avoid cell death or cell cycle arrest by prolonged Wg signaling ${ }^{47-49}$. Indeed, expression of Wg-mycCTD or untagged Wg-CTD for ten hours increased wing size by
17.6 and $31.3 \%$, respectively ( $n=31$, Fig. 4 f, g). Likewise, the number of PH3-positive cells was increased along the $p t c$ region compared to control $p t c>G F P$ discs (Fig. $4 \mathrm{~h}-\mathrm{j})$. Therefore, Wg-CTD is able to promote cell proliferation.

\section{Wg-CTD rescues the loss of sona phenotypes}

Wg-CTD would rescue the loss-of-function phenotypes of extracellular Sona if generation of Wg-CTD is a main function of extracellular Sona. To test this, sona RNAi$1^{11-4}$ (sona RNAi, hereafter) was coexpressed with $\mathrm{Wg}$ mycCTD and the wing phenotype and lethality were compared to those of sole sona RNAi expression ${ }^{10}$. All $n u b>$ sona $R N A i$ wings were small, wrinkled or both ${ }^{10}(n$ $=95$, Fig. $4 \mathrm{k}, \mathrm{m})$ but $68 \%$ of $n u b>$ sona $R N A i$, wg$m y c C T D$ wings were normal $(n=109$, Fig. $4 n, s)$. Notched wing phenotype was observed in $55 \%$ of $w g>$ sona $R N A i$ $(n=71)$ but only in $6 \%$ of $w g>w g-m y c C T D$, sona $R N A i$ $(n=68)$ flies (Fig. 4o-r). Furthermore, notched wing phenotype of $p t c>w g-m y c C T D$ was rescued in $p t c>s o n a$ $R N A i$, wg-mycCTD flies (Supplemental Fig. S8E-G). Therefore, Wg-CTD expression rescued loss of sona phenotypes.

\section{Wg-CTD activates canonical $\mathbf{W g}$ signaling}

Cytoplasmic Arm becomes stabilized by activation of canonical Wg signaling ${ }^{50}$. Because Wg-CTD expression rescued the lethality and wing defects induced by arm $R N A i$, Sgg or $d T C F^{D N}$ expression (Fig. 5a-p), we tested whether Wg-CTD stabilizes the cytoplasmic Arm. In fact, $c i>G F P$, wg-mycCTD wing discs had the increased level of Arm in the anterior region (Fig. 5q, r). More Arm was also present in the CX of $n u b>w g-m y c C T D$ compared to control nub-Gal4 wing discs (Fig. 5s).

We next examined whether Wg-CTD increases levels of Wg effector proteins, $\mathrm{Vg}$ and Dll that are induced by canonical Wg signaling ${ }^{51-54}$. Transient expression of WgCTD by en-Gal4 increased the level of Vg and Dll in the posterior region of wing discs (Fig. 6a-d), and Wg-CTDexpressing flp-out clones had higher level of Dll (Fig. 6e-g). The untagged Wg-CTD expression in en > $w g$-CTD discs also increased the level of Dll (Supplemental Fig. S9B). GFP-Wg-NTD and untagged Wg-NTD failed to change the level of Wg effector proteins (Fig. 6h, i and Supplemental Fig. S9A). Therefore, Wg-CTD is a new form of active Wg that induces canonical Wg signaling.

\section{Wg-CTD upregulates the level of Cyc D for cell proliferation}

Overexpressed $\mathrm{Wg}^{\mathrm{FL}}$ partially rescued the lethal phenotype of $w g$ mutants but Wg-CTD did not (Supplemental Fig. 9C-E). This demonstrates that Wg-CTD can carry out only subsets of Wg signaling and so called 'Wg signaling' is induced by combined activity of both $\mathrm{Wg}^{\mathrm{FL}}$ 


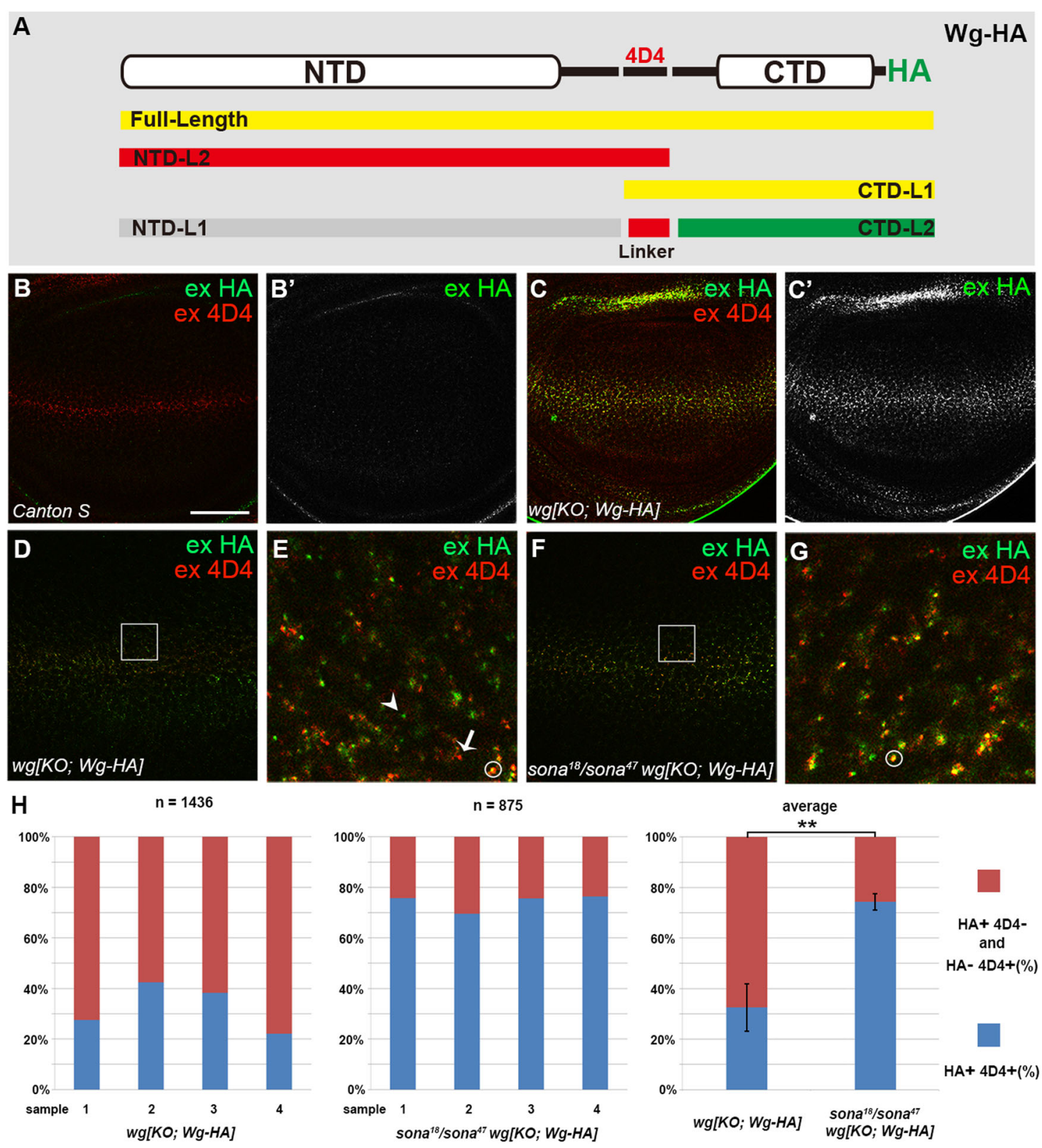

Fig. 3 Cleaved Wg fragments are present in the basal ECM of wing discs. a Domain structures of Wg-HA and cleaved products drawn with red, green, and yellow bars. The Grey bar indicates undetectable NTD ${ }^{\mathrm{L} 1}$ fragment. The 4D4 epitope is present in the linker region. The HA (green) and 4D4 (red) signals match with the colors in the confocal images. b, c Extracellular staining of a homozygous wg[KO;Wg-HA] wing disc at the basal ECM. In the $w g[K O ; W g-H A]$ strain, the endogenous $w g$ gene is replaced by a wg-HA transgene that fully supports fly development ${ }^{41}$. Extracellular Wg-HA was detected by anti-4D4 and anti-HA antibodies. Extracellular HA signals were detected only in wg[KO; Wg-HA] (c) but not in CS wing discs (b). These two

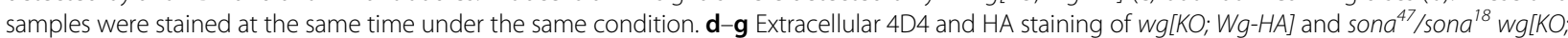
Wg-HA] wing discs. The son ${ }^{18}$ and son $a^{47}$ encode Sona with an internal deletion and a C-terminal truncation, respectively, and both cause pupal lethality ${ }^{10}$. Representative green $\left(\mathrm{HA}^{+} 4 \mathrm{D} 4^{-}\right.$including $\left.\mathrm{CTD}^{\mathrm{L2}}\right)$, red $\left(\mathrm{HA}^{-} 4 \mathrm{D} 4^{+}\right.$including $\mathrm{NTD}^{\mathrm{L} 2}$ or the linker fragment), and yellow $\left(\mathrm{HA}^{+} 4 \mathrm{D} 4^{+}\right.$ including $\mathrm{Wg}^{\mathrm{FL}}$ or $\mathrm{CTD}^{\mathrm{L} 1}$ ) signals are marked by an arrowhead, an arrow, and a circle, respectively. The squared regions in (d) and (f) are magnified in (e) and $(\mathbf{g})$, respectively. $\mathbf{h}$ The proportions of blue bars representing $\mathrm{HA}^{+} 4 \mathrm{D} 4^{+}$structures and red bars representing both $\mathrm{HA}^{+} 4 \mathrm{D}^{-}$and $\mathrm{HA}^{-} 4 \mathrm{D} 4^{+}$ structures. $4 \mathrm{wg}[\mathrm{KO} ; \mathrm{Wg}-\mathrm{HA}]$ discs and 4 son $^{47} / \mathrm{sona}^{18} \mathrm{wg}[\mathrm{KO} ; \mathrm{Wg}-\mathrm{HA}]$ discs were used for counting extracellular structures. The total numbers of counted structures are written on the top of the graph. The average percentages of counted vesicles are shown on the right. Graphs are displayed as mean \pm S.E.M, where ${ }^{* *} p<0.01$. Scale bars for (b) and (c), $60 \mu \mathrm{m}$; (d) and (f), $40 \mu \mathrm{m}$; (e) and (g), $5 \mu \mathrm{m}$ 

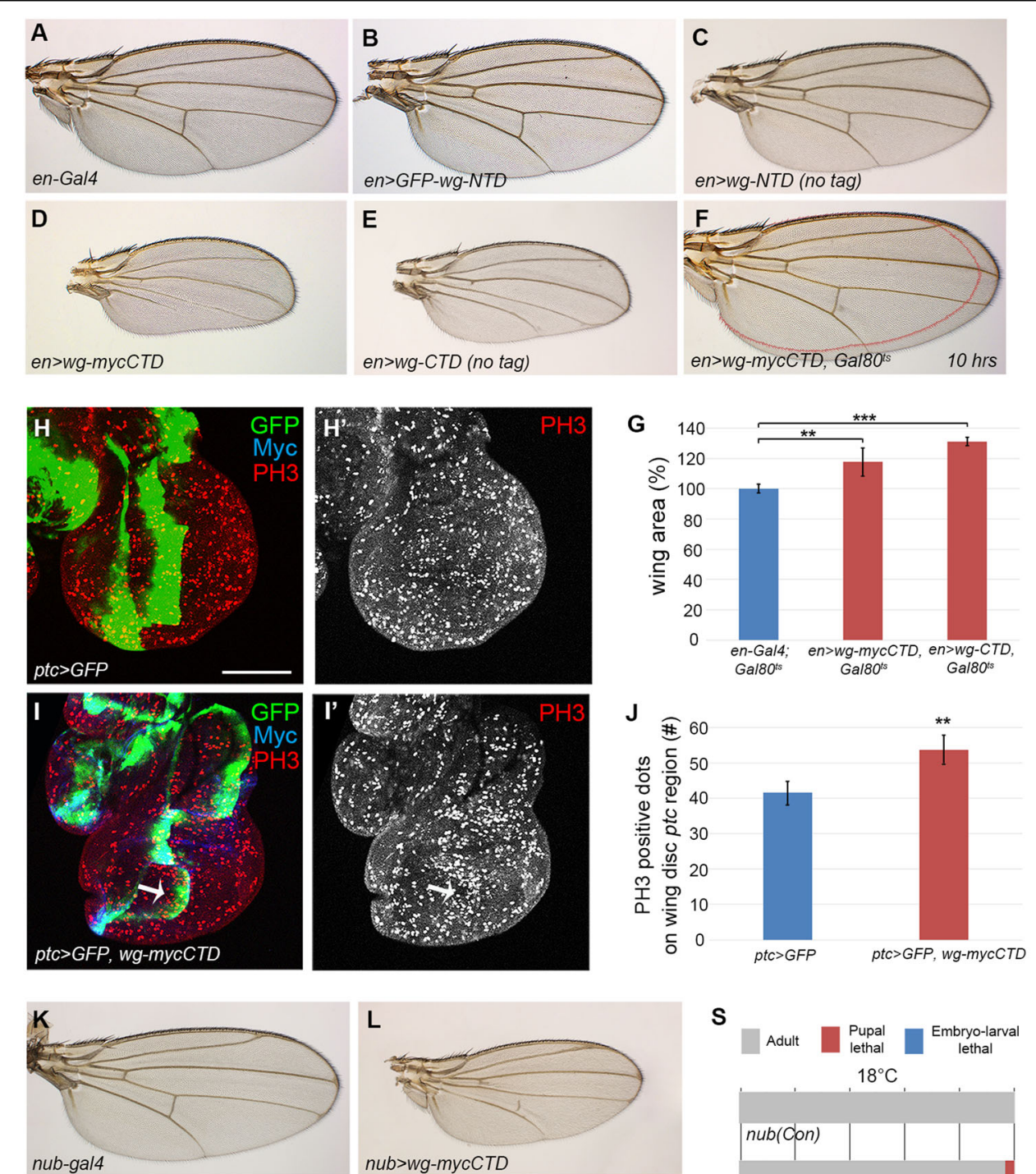

S
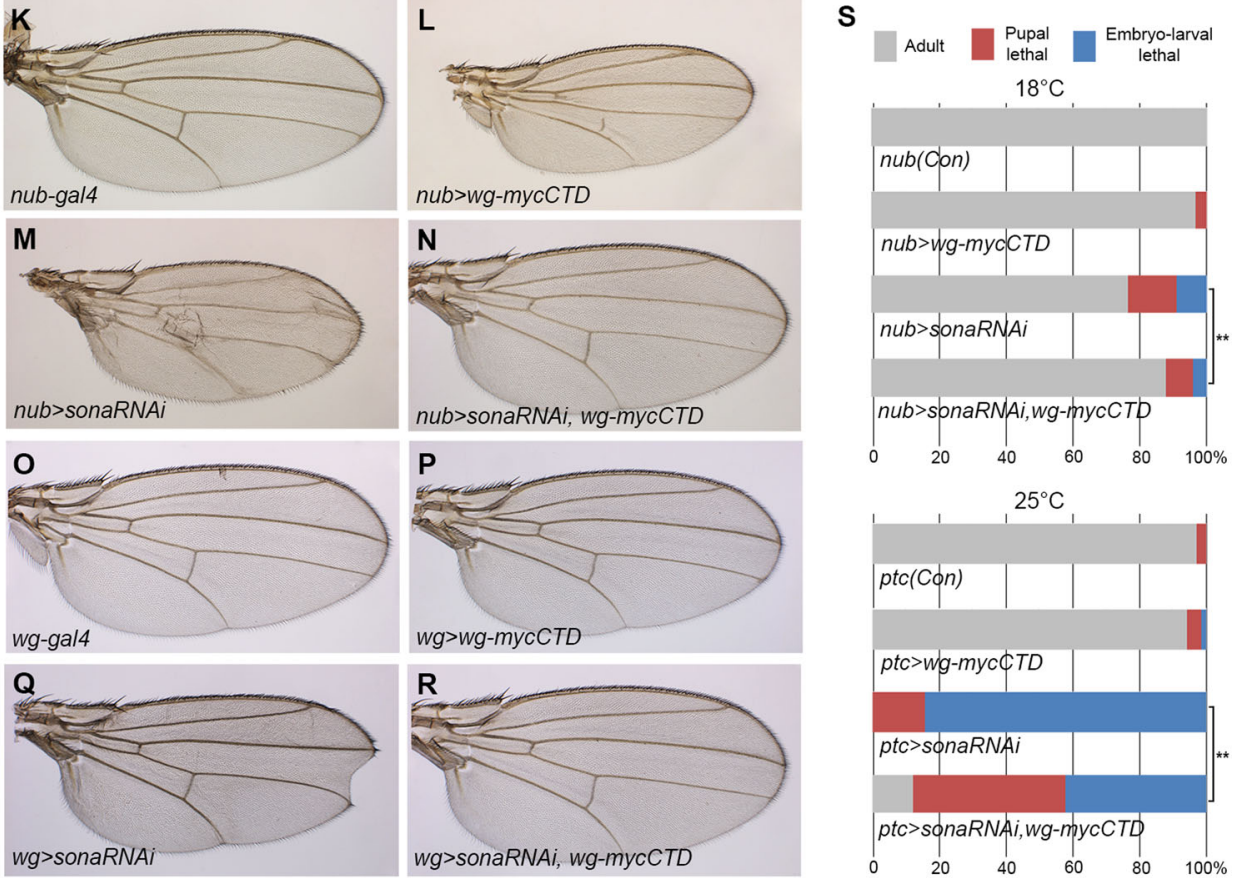

Fig. 4 (See legend on next page.) 
(see figure on previous page)

Fig. $4 \mathbf{~ W g}$-CTD but not Wg-NTD is functional in vivo. The genotypes of female wings are indicated at the lower left of each panel. a The en-Gal4 wing at $18^{\circ} \mathrm{C}$ as a control. b, c Both en $>$ GFP-wg-NTD wing in (b) and en $>$ wg-NTD (no tag) wing in (c) were normal. d, e prolonged expressions of Wg-CTD in en > wg-mycCTD flies (d) and en > wg-CTD (no tag) flies (e) induce deformed wings with smaller posterior region than the control wing in (a) similar to phenotypes by prolonged expression of $\mathrm{Wg}^{48,49}$. $\mathbf{f}$ en $>$ wg-mycCTD, Gal80 ts wings from flies cultured for $10 \mathrm{~h}$ at $30^{\circ} \mathrm{C}$ during the midthird instar stage for transient Wg-mycCTD expression. Outline of the control wing in (a) is drawn in (f). $\mathbf{g}$ The average wing size of en $>$ wg-mycCTD,

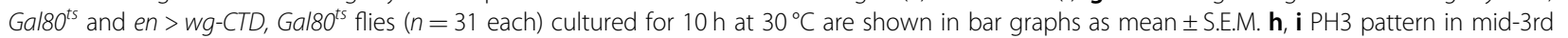
instar wing discs of ptc > GFP and ptc $>$ GFP, wg-mycCTD. An arrow indicates increase in PH3 staining along the ptc region in (i). $\mathbf{j}$ The graph represents the average number of PH3 positive signals in the ptc region of ptc $>$ GFP and ptc > GFP, wg-mycCTD wing discs $(n=10)$. $\mathbf{k}-\mathbf{r}$ Wg-mycCTD expression rescues sona RNAi phenotypes. All nub > sona RNAi wings are small and malformed in (m). Wing phenotypes of nub $>$ sona RNAi were rescued by coexpression of Wg-mycCTD in (n). wg $>$ wg-myc-CTD wings in (p) did not show any phenotype similar to the control wg-Gal4 wings in (o). wg > sona RNAi $(n=71)$ had notched wing phenotype in $(\mathbf{q})$, but wg > sona RNAi, wg-mycCTD had normal wings $(n=68)$ in $(\mathbf{r})$. $\mathbf{s}$ Wg-mycCTD expression partially suppressed the lethality induced by sona RNAi. Adult flies, pupal lethal and embryonic to larval lethal in percentages are drawn in colored bars. $23 \%$ of nub > sona RNAi flies were embryonic to pupal lethal, but only $12 \%$ of nub > sona RNAi, wg-mycCTD flies were embryonic to pupal lethal $(n=160$ each). Similarly, none of ptc $>$ sona RNAi flies but $12 \%$ of ptc $>$ sona RNAi, wg-mycCTD flies $(n=140)$ survived to adulthood and their lethal stage was also significantly delayed from embryonic to pupal stage (Supplemental Fig. S7A-D). Scale bar, $100 \mu$ m, $* * p<0.01 ; * * * p<0.001$

and cleaved Wg-CTD. An important question is then whether Wg-CTD has any unique functions unshared by $\mathrm{Wg}^{\mathrm{FL}}$. We hypothesized that Wg-CTD may be more specialized for cell proliferation than $\mathrm{Wg}^{\mathrm{FL}}$ because Sona increases the level of Cyc $\mathrm{D}^{13}$. Indeed, Cyc D was upregulated by prolonged expression of Wg-CTD in the anterior region of $c i>w g-m y c C T D$ wing discs and by transient expression of Wg-CTD in the posterior region of en $>w g-m y c C T D$, Galso ${ }^{t s}$ discs cultured for $12 \mathrm{~h}$ at $30^{\circ} \mathrm{C}$ (Fig. 7a, b; Supplemental Fig. S9F, G). GFP-Wg ${ }^{\mathrm{FL}}$ or GFP-Wg-NTD expression, however, did not change the level of Cyc D (Fig. 7c, d). Taken together, Wg-CTD is able to induce Cyc D. Wg signaling plays an important role in neuronal differentiation by inducing sens in the DV margin of wing discs ${ }^{55-57}$. Transient expression of GFPWg increased the level of Sens in ptc $>G F P$-wg, Gal80 ${ }^{t s}$ discs, and induced ectopic sensory bristles in $n u b>G F P$ $w g$, Gal80 ${ }^{t s}$ wings, which are consistent with previous reports $^{58,59}$ (Fig. 7e-h). In contrast, transient expression of Wg-CTD did not induce ectopic Sens in $p t c>w g$ $m y c C T D$ Galso $^{t s}$ and $e n>w g$-mycCTD Gal8o ${ }^{t s}$ wing discs (Fig. 7i and Supplemental Fig. S9I). Wings of $n u b>w g$ $m y c C T D$, Gal80 ${ }^{t s}$, nub > wg-mycCTD, and $n u b>w g-N T D$ flies also had no ectopic bristles (Fig. 7j and Supplemental Fig. S9K-L). Thus, Wg-CTD is not able to induce Sens unlike $\mathrm{Wg}^{\mathrm{FL}}$.

\section{Discussion}

We report here that Sona cleaves extracellular Wg into Wg-NTD and Wg-CTD, and the Wg-CTD is a new form of active Wg (Supplemental Fig. S10). Because Wg-CTD substantially rescued the sona loss-of-function phenotypes such as lethality and wing defects (Fig. 4), generation of Wg-CTD seems to be one of Sona's major functions. Wnt modifications such as lipidation and glycosylation have been extensively studied, but Wnt cleavage has not been addressed except for the Xenopus Tiki protease. Tiki reduces Wnt secretion by cleaving the amino-terminal region of intracellular Wnt that is required for the lipidation of $\mathrm{Wnt}^{60}$. While Tiki aims to decrease the amount of secreted Wnt, Sona aims to generate a new active form of $\mathrm{Wg}$ from an already active $\mathrm{Wg}^{\mathrm{FL}}$.

Genetic interaction between $w g-C T D$ and other Wg signaling components indicates that Wg-CTD activates $\mathrm{Wg}$ signaling similar to $\mathrm{Wg}^{\mathrm{FL}}$ (Fig. 5). However, there are several differences between these two forms of Wg. First, Wg-CTD but not Wg ${ }^{\mathrm{FL}}$ increased the level of Cyc D (Fig. 7). Overexpressed Cyc D-Cdk4 in flies accelerates cell division of undifferentiated cells such as wing disc cells ${ }^{61}$. Sona also induces Cyc D and promotes cell proliferation in a cell non-autonomous manner ${ }^{13}$. Therefore, Wg-CTD generated by extracellular Sona seems to induce Cyc D in the neighboring cells for cell proliferation. Second, both $\mathrm{Wg}_{-C T D}{ }^{\mathrm{L} 1}$ and $\mathrm{Wg}-\mathrm{CTD}^{\mathrm{L} 2}$ are less stable than $\mathrm{Wg}^{\mathrm{FL}}$. Instability of Wg-CTD may be an essential feature because mitogens and their downstream components are often removed by degradation to prevent excessive cell

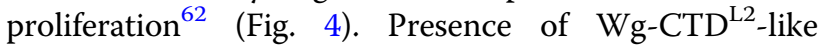
structures in wing discs (Fig. 3), however, implies that these $\mathrm{Wg}$-CTD ${ }^{\mathrm{L} 2}$-like structures may be stabilized in vivo by ECM components to achieve spatiotemporal regulation of the mitogenic activity ${ }^{63,64}$. Third, Wg-CTD is not able to induce Sens (Fig. 7 and Supplemental Fig. S8). Sens expression in the DV midline is required for differentiation of wing margin bristles ${ }^{55-57}$, unlike $\mathrm{Vg}$ that is essential for cell proliferation and cell survival ${ }^{23,65}$.

The difference between the two Wg forms in Sens induction may be due to their differential affinity to $\mathrm{Fz}$ receptors, based on the report that NTD and CTD of vertebrate Wnts able to interact $\mathrm{Fz}$ receptors independently from each other with different affinity ${ }^{66}$. It has been proposed that Wnt is generated during evolution via the fortuitous fusion of two ancestral proteins analogous to its NTD, homologous to a class of lipid-interacting proteins, and CTD, homologous to a group of cytokines involved in cell signaling ${ }^{42,66,67}$. This explains why NTD mutants are unable to be secreted ${ }^{68}$, while CTD mutants are secreted but inactive ${ }^{69}$. Given the evolutionary conservation of the 


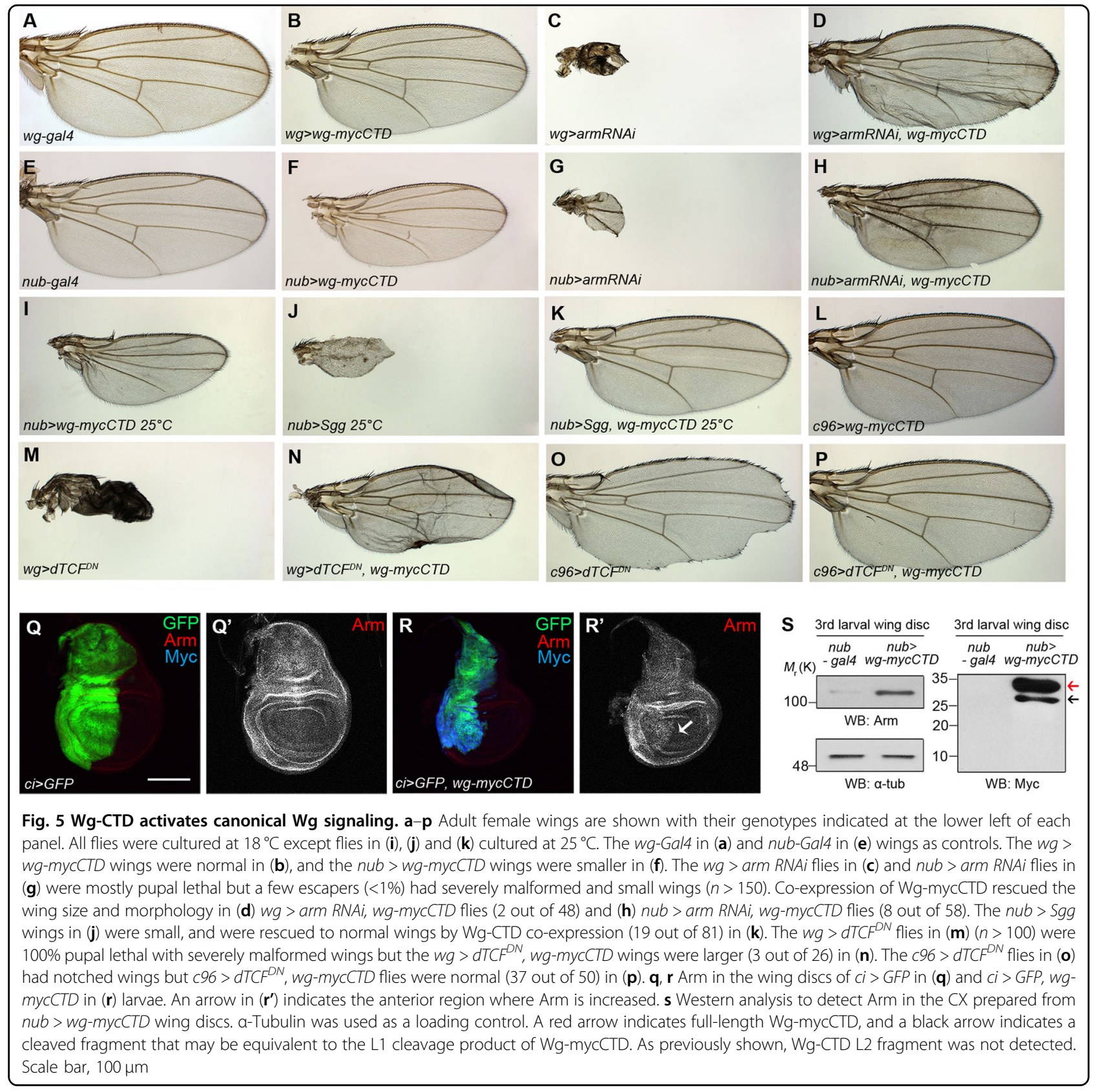

components of Wnt signaling, ADAMTSs may also be involved in the generation of functional Wnt-CTD in mammals ${ }^{70,71}$. We expect that further study on the relationship between Wnts and ADAMTSs will expand our understanding on Wnt signaling and Wnt-related diseases.

\section{Materials and methods}

\section{Drosophila strains, transgenic lines and generation of} ectopic clones

sona mutants, sona RNAi lines, UAS-sona, and UASsona-HA are described elsewhere ${ }^{10}$. The UAS-wg- $m y c C T D, U A S-G F P-w g-N T D, U A S-w g-C T D$ and $U A S-w g-$ NTD flies were generated for this study. UAS-CD63$G F P^{72}, U A S-G F P-w g^{73}, w g[K O ; W g-H A]^{41}, U A S-G F P-$ lamp $p^{74}, w g^{\text {Gal } 475}$ and $c i-G a l 4^{76}$ were kindly provided by other labs that produced them. All other lines were obtained from the Bloomington stock center.

\section{DNA constructs}

The $p A c-G F P-w g$ and $p A c-w g-3 X H A$ were constructed by recombining the $p A c 5.1$ vector with GFP-wg or $w g$ $3 X H A$ obtained from $M K 33-G F P-w g$ (a gift from J.P. Vincent, unpublished) or $U A S-w g-3 X H A^{37}$. To generate 

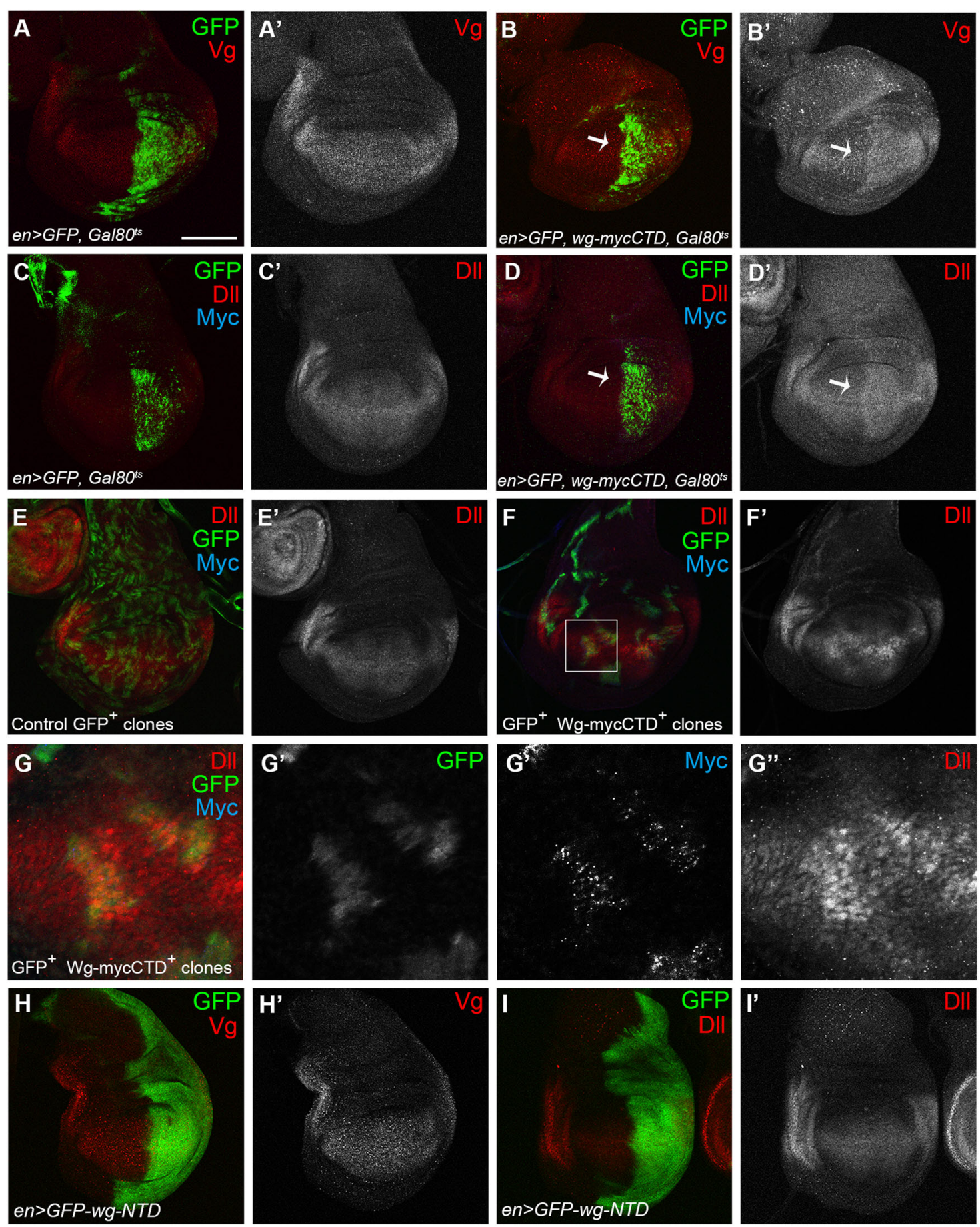

Fig. $6 \mathbf{~ W g}$-CTD increases the level of DII and $\mathbf{~ g g . ~ a - d ~ T h e ~ f l i e s ~ w e r e ~ s h i f t e d ~ f r o m ~} 18^{\circ} \mathrm{C}$ to $30^{\circ} \mathrm{C}$ for $10 \mathrm{~h}$ during the late second and early third larval

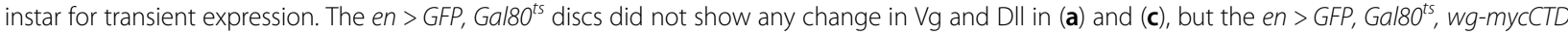
discs showed increased $\mathrm{Vg}$ and DIl in the posterior region marked by arrows in (b) and (d). e- $\mathbf{g}$ Analysis of GFP ${ }^{+}$clones that overexpress Wg-CTD in hsflp; actin >y > Gal4; UAS-GFP (e) and hsflp; actin >y > Gal4; UAS-GFP/UAS-wgmycCTD (f) flies. GFP ${ }^{+}$control clones in (e) showed no change but $\mathrm{GFP}^{+} \mathrm{Wg}-\mathrm{CTD}^{+}$clones in $(\mathbf{f})$ had higher level of Dll. The boxed region in $(\mathbf{f})$ is magnified in $(\mathbf{g})$. $\mathbf{h}$, $\mathbf{i}$ en $>$ GFP-wg-NTD wing discs showed no change in the levels of $\mathrm{Vg}$ in (h) or DII in (i). Scale bar, $100 \mu \mathrm{m}$ except (g), $40 \mu \mathrm{m}$ 

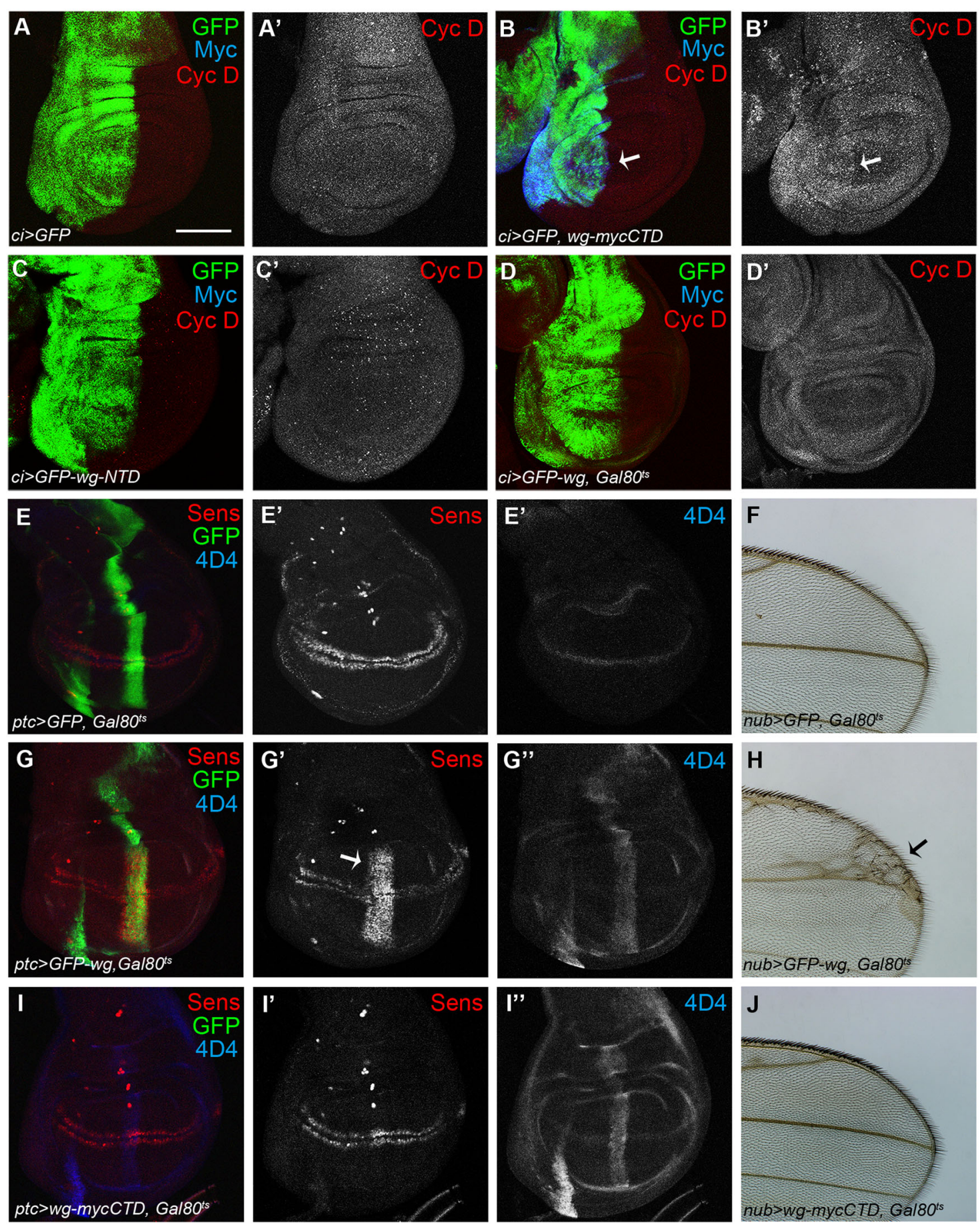

Fig. 7 Wg-CTD may be specialized for cell proliferation than differentiation. Transgenes were transiently expressed by shifting the culture temperature to $30^{\circ} \mathrm{C}$ for $12 \mathrm{~h}$ during the late second and early third larval instar in all cases. a-d The level of Cyc D in wing discs. Arrows mark the region with higher level of Cyc D in en > GFP, wg-mycCTD discs in (b). No changes in control ci > GFP in (a), en > GFP-wg-NTD in (c) and en > GFP-Wg, Gal80 $0^{t s}$ in (d) discs. e-j Sens expression in wing discs and sensory bristle formation in adult wings. Sens level in control (e) and ptC $>$ wg-mycCTD, Galsots wing discs in (i). The arrow indicates ectopic Sens by GFP-Wg ${ }^{\mathrm{FL}}$ in ptc $>$ GFP-wg, Galsots wing discs in (g). Expression of full-length GFP-Wg in (h) induced ectopic bristles in wing blades, but control in (f) and Wg-CTD expression in (j) did not induce any ectopic bristles. Scale bar, $100 \mu \mathrm{m}$ 
the GFP-wg-NTD (GFP-NTD) and $w g-m y c C T D$ constructs, a $m y c$ tag was inserted in the DNA corresponding to the region between Arg367 and Tyr368 in GFP-Wg. DNA fragments representing GFP-NTD (1-245) and mycCTD (1-22, 245-468) were then amplified by PCR and inserted into pUAST vectors by recombination cloning methods.

\section{Cell lines, cell culture, and exosome preparation}

Drosophila S2 tub-wg, S2R+, and S2 cell lines were obtained from DGRC. S2 GFP-wg, wg-3XHA, and sona$H A$ stable cell lines were generated by selection under $2.5 \mu \mathrm{g} / \mathrm{ml}$ hygromycin B (Invitrogen) as follows. Drosophila S2 cell were grown in M3 media (Sigma-Aldrich) supplemented with $10 \%$ IMS (Sigma-Aldrich) at $25^{\circ} \mathrm{C}$. Stable cell lines were grown with hygromycin in $10 \%$ IMS M3 media, and S2 tub-wg cells were cultured in 10\% FBS M3 media. Transfections were carried out with Effectene (Qiagen) or Cellfectin (Invitrogen) according to the manufacturers' instructions. For exosome preparation, 7-40 ml of conditioned media obtained from cultures $\left(1.25 \times 10^{6}\right.$ cells $\left./ \mathrm{ml}\right)$ were used as described ${ }^{19}$. The size and number of the exosomes in the P100 fraction were measured by Nanosight NC300 (Malvern Instruments).

\section{Immunocytochemistry and Western analysis}

Fly larvae were cultured at $25^{\circ} \mathrm{C}$ unless stated otherwise. Wing discs from the late third instar larvae were used for intracellular staining and extracellular staining ${ }^{24}$. For immunocytochemistry, we used Sona-Pro, 1:300-500; Golgi (Calbiochem, mouse), 1:200; GFP (Abd serotec, sheep), 1:100; Senseless (a gift from H. Bellen, guinea pig), 1:1000; HRS (a gift from H. Bellen, guinea pig), 1:1000; WgN (sc-28646 Santa Cruz, rabbit), 1:100; Wg (DSHB, mouse), 1:1000; HA (Roche, rat), 1:300; HA (Santa Cruz, rabbit), 1:300; Vg (gift from Sean B. Carroll, rabbit), 1:100; Wg (DSHB, mouse), 1:100; Dll (Santa Cruz, goat), 1:100. For the extracellular staining of proteins, we used 10 times more antibodies than for the intracellular staining. Fluorescent images were captured using a Zeiss LSM laser scanning confocal microscope and processed with Adobe Photoshop.

Western analysis was carried out as described ${ }^{10}$. For western analysis, we used Sona-Pro (our lab, rabbit), 1:5000; HA (Santa Cruz, rabbit), 1: 250; GFP (Abcam, rabbit), 1:10,000; Wg (DSHB, mouse), 1:500-1000; Syntaxin 1A (DSHB, mouse), 1:25; Alix (gift from T. Aigaki, mouse), 1:500; Actin (DSHB, mouse), 1:500; Calnexin (gift from N.J. Colley, rabbit), 1:2000.

\section{Electron microscopy}

For immunogold labeling, P100 fraction from S2 sona$H A$ cells were plated on grids, blocked with 5\% BSA in PBS and incubated with anti-HA antibody (1:5). Then, samples were washed with $0.1 \%$ BSA in PBS and incubated in secondary anti-rabbit antibody conjugated with $15 \mathrm{~nm}$ gold particles (AURION). After 8 times wash with PBS for 5 min each, samples were incubated in $1 \%$ glutaraldehyde for $5 \mathrm{~min}$. Then, samples were washed with $\mathrm{H}_{2} \mathrm{O}$ for 8 times before staining with Phosphotungstic acid (PTA). Sample grids were air-dried completely and visualized using a transmission electron microscope (Talos F200X).

\section{In vitro GST-Wg cleavage assay}

For purification of GST-Wg, pGEX-4T-1-WgCterm was expressed in BL21 E. coli strain. Then, we purified the GST-Wg protein by standard column-based protocols (GST-column, $1^{\text {st }}$ SP Sepharose column, $2^{\text {nd }}$ SP Sepharose column). For purification of active Sona from S2 cell culture, $\mathrm{CX}$ and $\mathrm{SN}_{\Delta}$ fractions were prepared and lysis buffer without EDTA and Protein inhibitor cocktail (PIC) were added to these fractions. Active Sona was obtained by mixing with HA-conjugated bead and precipitating the beads. GST-Wg and active Sona were mixed and incubated at $25^{\circ} \mathrm{C}$ overnight.

\section{Sucrose step gradient}

Exosome pellets were resuspended in $0.25 \mathrm{M}$ sucrose and loaded on top of a sucrose step gradient before being centrifuged at 100,000xg in a Beckman SW41Ti rotor for $3 \mathrm{~h}$ as described ${ }^{77}$. Ten to twelve fractions of $1 \mathrm{~mL}$ each were then manually collected from the bottom of the gradient.

\section{Wg reporter assay}

The Wg reporter assay was carried out by conventional methods. WISIR vector that contains both firefly luciferase under the control of a Wg-responsive promoter and Renilla luciferase under the control of a Copia promoter was transfected into S2R+ cells. After one day of culture, cells were splitted to a 48 well plate and incubated for $3 \sim 4 \mathrm{~h}$ until the experimental treatment started. After $24 \mathrm{~h}$ of treatment, cells were lysed by following the manufacturer's instructions of the Dual-Luciferase Repoter Assay System (Promega). Each condition was tested in triplicate.

\section{Acknowledgements}

We thank K.W. Choi and K.H. Kang for their critical reading of this manuscript We also thank Munashingha P. R. for preparation of GST-Wg protein. We are indebted to J. P. Vincent, K. Basler, S. Eaton, S. Hayashi, S. M. Cohen, L. S. Shashidhara, R. Holmgren, and H. Kramer for fly lines, to K. Basler, J. P. Vincent, and S.T. Hong for DNA constructs, and to H. Bellen, N.J. Colley, T. Aigaki and Sean B. Carroll for antibodies. We thank Bloomington Stock Center, Drosophila Genetic Resource Center, and Developmental Studies Hybridoma Bank for fly strains and antibodies. This research was supported by grants from the National Research Foundation of Korea (NRF-2017R1A2B4009254 and NRF2019R1H1A2039726) and National Research Council of Science and Technology (DRC-14-KRISS). 


\section{Conflict of interest}

The authors declare that they have no conflict of interest.

\section{Publisher's note}

Springer Nature remains neutral with regard to jurisdictional claims in published maps and institutional affiliations.

Supplementary Information accompanies this paper at (https://doi.org/ 10.1038/s41419-019-1794-8).

Received: 27 July 2018 Revised: 14 June 2019 Accepted: 4 July 2019 Published online: 22 July 2019

\section{References}

1. Bonnans, C., Chou, J. \& Werb, Z. Remodelling the extracellular matrix in development and disease. Nat. Rev. Mol. Cell Biol. 15, 786-801 (2014).

2. Apte, S. S. \& Parks, W. C. Metalloproteinases: A parade of functions in matrix biology and an outlook for the future. Matrix Biol. 44-46, 1-6 (2015).

3. Mott, J. D. \& Werb, Z. Regulation of matrix biology by matrix metalloproteinases. Curr. Opin. Cell Biol. 16, 558-564 (2004).

4. Rocks, N. et al. Emerging roles of ADAM and ADAMTS metalloproteinases in cancer. Biochimie 90, 369-379 (2008).

5. Porter, S., Clark, I. M., Kevorkian, L. \& Edwards, D. R. The ADAMTS metalloproteinases. Biochem J. 386, 15-27 (2005).

6. Kelwick, R., Desanlis, I., Wheeler, G. N. \& Edwards, D. R. The ADAMTS (A Disintegrin and Metalloproteinase with Thrombospondin motifs) family. Genome Biol. 16, 113 (2015).

7. Le Goff, C. \& Cormier-Daire, V. The ADAMTS(L) family and human genetic disorders. Hum. Mol. Genet. 20, 163-167 (2011).

8. El, HourM. et al. Higher sensitivity of Adamts12-deficient mice to tumor growth and angiogenesis. Oncogene 29, 3025-3032 (2010).

9. Rocks, N. et al. ADAMTS-1 metalloproteinase promotes tumor development through the induction of a stromal reaction in vivo. Cancer Res. $\mathbf{6 8}, 9541-9550$ (2008).

10. Kim, G. W. et al. Sol narae (Sona) is a Drosophila ADAMTS involved in Wg signaling. Sci. Rep. 6, 31863 (2016).

11. Banziger, C. et al. Wntless, a conserved membrane protein dedicated to the secretion of Wnt proteins from signaling cells. Cell 125, 509-522 (2006).

12. Bartscherer, K., Pelte, N., Ingelfinger, D. \& Boutros, M. Secretion of Wnt ligands requires Evi, a conserved transmembrane protein. Cell 125, 523-533 (2006).

13. Tsogtbaatar, O. et al. An ADAMTS Sol narae is required for cell survival in Drosophila. Sci. Rep. 9, 1270 (2019).

14. Baldin, V., Lukas, J., Marcote, M. J., Pagano, M. \& Draetta, G. Cyclin D1 Is a Nuclear-Protein Required for Cell-Cycle Progression in G(1). Gene Dev. 7, 812-821 (1993).

15. Sharma, R. P. Wingless - a new mutant in D. melanogaster. Drosoph. Inf. Serv. 50, 134 (1973).

16. Rijsewijk, F. et al. The Drosophila homolog of the mouse mammary oncogene int- 1 is identical to the segment polarity gene wingless. Cell 50, 649-657 (1987).

17. Mann, M. C. The occurrence and hereditary behavior of two new dominant mutations in an inbred strain of Drosophila melanogaster. Genetics 8, 27-36 (1923).

18. Port, F. \& Basler, K. Wnt trafficking: new insights into Wnt maturation, secretion and spreading. Traffic 11, 1265-1271 (2010).

19. Gross, J. C., Chaudhary, V., Bartscherer, K. \& Boutros, M. Active Wnt proteins are secreted on exosomes. Nat. Cell Biol. 14, 1036-1045 (2012).

20. Gross, J. C. \& Boutros, M. Secretion and extracellular space travel of Wht proteins. Curr. Opin. Genet. Dev. 23, 385-390 (2013).

21. Bejsovec, A. Wingless/Wnt signaling in Drosophila: the pattern and the pathway. Mol. Reprod. Dev. 80, 882-894 (2013).

22. Nusse, R. \& Clevers, H. Wnt/beta-catenin signaling, disease, and emerging therapeutic modalities. Cell 169, 985-999 (2017).

23. Delanoue, $\mathrm{R}$. et al. The Drosophila wing differentiation factor VestigialScalloped is required for cell proliferation and cell survival at the dorso-ventral boundary of the wing imaginal disc. Cell Death. Differ. 11, 110-122 (2004).
24. Strigini, M. \& Cohen, S. M. Wingless gradient formation in the Drosophila wing Curr. Biol. 10, 293-300 (2000)

25. Neumann, C. J. \& Cohen, S. M. A hierarchy of cross-regulation involving Notch, wingless, vestigial and cut organizes the dorsal/ventral axis of the Drosophila wing. Development 122, 3477-3485 (1996).

26. Ryoo, H. D., Gorenc, T. \& Steller, H. Apoptotic cells can induce compensatory cell proliferation through the JNK and the wingless signaling pathways. Dev. Cell 7, 491-501 (2004).

27. Angers, S. \& Moon, R. T. Proximal events in Wnt signal transduction. Nat. Rev. Mol. Cell Biol. 10, 468-477 (2009).

28. Shtutman, M. et al. The cyclin D1 gene is a target of the beta-catenin/LEF-1 pathway. Proc. Natl. Acad. Sci. USA 96, 5522-5527 (1996).

29. He, T. C. et al. Identification of c-MYC as a target of the APC pathway. Science 281, 1509-1512 (1998).

30. Koles, K. \& Budnik, V. Wnt signaling in neuromuscular junction development. Cold Spring Harb. Perspect. Biol. 4, pii: a008045 (2012).

31. Herr, P., Hausmann, G. \& Basler, K. WNT secretion and signalling in human disease. Trends Mol. Med. 18, 483-493 (2012).

32. Clevers, H. \& Nusse, R. Wnt/beta-catenin signaling and disease. Cell 149, 1192-1205 (2012)

33. Beckett, K. et al. Drosophila S2 cells secrete wingless on exosome-like vesicles but the wingless gradient forms independently of exosomes. Traffic 14, 82-96 (2013).

34. Koles, K. et al. Mechanism of evenness interrupted (Evi)-exosome release at synaptic boutons. J. Biol. Chem. 287, 16820-16834 (2012).

35. Willert, K. \& Nusse, R. Wnt proteins. Cold Spring Harb. Perspect. Biol. 4, a007864 (2012).

36. Brook, W. J. \& Cohen, S. M. Antagonistic interactions between wingless and decapentaplegic responsible for dorsal-ventral pattern in the Drosophila Leg. Science 273, 1373-1377 (1996).

37. Port, F. et al. Wingless secretion promotes and requires retromer-dependent cycling of Wntless. Nat. Cell Biol. 10, 178-185 (2008).

38. Casares, F. \& Mann, R. S. A dual role for homothorax in inhibiting wing blade development and specifying proximal wing identities in Drosophila. Developmen 127, 1499-1508 (2000).

39. Phillips, R. G. \& Whittle, J. R. wingless expression mediates determination of peripheral nervous system elements in late stages of Drosophila wing disc development. Development 118, 427-438 (1993).

40. Couso, J. P., Bishop, S. A. \& Martinez Arias, A. The wingless signalling pathway and the patterning of the wing margin in Drosophila. Development $\mathbf{1 2 0}$ 621-636 (1994).

41. Baena-Lopez, L. A., Alexandre, C., Mitchell, A., Pasakarnis, L. \& Vincent, J. P. Accelerated homologous recombination and subsequent genome modification in Drosophila. Development 140,4818-4825 (2013).

42. von Maltzahn, J., Zinoviev, R., Chang, N. C., Bentzinger, C. F. \& Rudnicki, M. A. A truncated Wnt7a retains full biological activity in skeletal muscle. Nat. Commun. 4, 2869 (2013)

43. Yun, C. \& Dasgupta, R. Luciferase reporter assay in Drosophila and mammalian tissue culture cells. Curr. Protoc. Chem. Biol. 6, 7-23 (2014).

44. Baena-Lopez, L. A., Franch-Marro, X. \& Vincent, J. P. Wingless promotes proliferative growth in a gradient-independent manner. Sci. Signal. 2, 60 (2009).

45. Ren, F. et al. Hippo signaling regulates Drosophila intestine stem cell proliferation through multiple pathways. Proc. Natl. Acad. Sci. USA 107, 21064-21069 (2010)

46. Cordero, J. B., Stefanatos, R. K., Myant, K., Vidal, M. \& Sansom, O. J. Nonautonomous crosstalk between the Jak/Stat and Egfr pathways mediates Apc1-driven intestinal stem cell hyperplasia in the Drosophila adult midgut. Development 139, 4524-4535 (2012).

47. Johnston, L. A. \& Edgar, B. A. Wingless and Notch regulate cell-cycle arrest in the developing Drosophila wing. Nature 394, 82-84 (1998).

48. Singh, A., Shi, X. \& Choi., K. W. Lobe and Serrate are required for cell survival during early eye development in Drosophila. Development 133, 4771-4781 (2006).

49. Zhang, S. et al. The canonical Wg signaling modulates Bsk-mediated cell death in Drosophila. Cell Death Dis. 6, e1713 (2015).

50. Wu, C. H. \& Nusse, R. Ligand receptor interactions in the Wnt signaling pathway in Drosophila. J. Biol. Chem. 277, 41762-41769 (2002).

51. Diaz-Benjumea, F. J. \& Cohen, S. M. Serrate signals through Notch to establish a Wingless-dependent organizer at the dorsal/ventral compartment boundary of the Drosophila wing. Development 121, 4215-4225 (1995). 
52. Maves, L. \& Schubiger, G. A molecular basis for transdetermination in Drosophila imaginal discs: interactions between wingless and decapentaplegic signaling. Development 125, 115-124 (1998)

53. $\mathrm{Wu}, \mathrm{J}$. \& Cohen, S. M. Proximodistal axis formation in the Drosophila leg: subdivision into proximal and distal domains by Homothorax and Distal-less. Development 126, 109-117 (1999).

54. Neumann, C. J. \& Cohen, S. M. Long-range action of Wingless organizes the dorsal-ventral axis of the Drosophila wing. Development 124,871-880 (1997).

55. Baeg, G. H., Selva, E. M., Goodman, R. M., Dasgupta, R. \& Perrimon, N. The Wingless morphogen gradient is established by the cooperative action of Frizzled and Heparan Sulfate Proteoglycan receptors. Dev. Biol. 276, 89-100 (2004).

56. Parker, D. S., Jemison, J. \& Cadigan, K. M. Pygopus, a nuclear PHD-finger protein required for Wingless signaling in Drosophila. Development 129 2565-2576 (2002).

57. Nolo, R., Abbott, L. A. \& Bellen, H. J. Senseless, a Zn finger transcription factor, is necessary and sufficient for sensory organ development in Drosophila. Cell 102, 349-362 (2000).

58. Lunde, $\mathrm{K}$. et al. Activation of the knirps locus links patterning to morphogenesis of the second wing vein in Drosophila. Development 130, 235-248 (2003).

59. Jafar-Nejad, H., Tien, A. C., Acar, M. \& Bellen, H. J. Senseless and Daughterless confer neuronal identity to epithelial cells in the Drosophila wing margin. Development 133, 1683-1692 (2006).

60. Zhang, X. et al. Tiki1 is required for head formation via Wnt cleavage-oxidation and inactivation. Cell 149, 1565-1577 (2012).

61. Datar, S. A., Jacobs, H. W., de la Cruz, A. F., Lehner, C. F. \& Edgar, B. A. The Drosophila cyclin D-Cdk4 complex promotes cellular growth. EMBO J. 19 4543-4554 (2000).

62. Bouge, A. L. \& Parmentier, M. L. Tau excess impairs mitosis and kinesin-5 function, leading to aneuploidy and cell death. Dis. Model Mech. 9, 307-319 (2016).

63. Chang, Y. H. \& Sun, Y. H. Carrier of Wingless (Cow), a secreted heparan sulfate proteoglycan, promotes extracellular transport of Wingless. PLOS ONE 9, e111573 (2014).

64. Baeg, G. H., Lin, X., Khare, N., Baumgartner, S. \& Perrimon, N. Heparan sulfate proteoglycans are critical for the organization of the extracellular distribution of Wingless. Development 128, 87-94 (2001).
65. Baena-Lopez, L. A. \& Garcia-Bellido, A. Control of growth and positional information by the graded vestigial expression pattern in the wing of Drosophila melanogaster. Proc. Natl. Acad. Sci. USA 103, 13734-13739 (2006).

66. Janda, C. Y., Waghray, D., Levin, A. M., Thomas, C. \& Garcia, K. C. Structural basis of Wnt recognition by Frizzled. Science 337, 59-64 (2012).

67. Bazan, J. F., Janda, C. Y. \& Garcia, K. C. Structural architecture and functional evolution of Wnts. Dev. Cell. 23, 227-232 (2012).

68. Tang, $\mathrm{X}$. et al. Roles of $\mathrm{N}$-glycosylation and lipidation in $\mathrm{Wg}$ secretion and signaling. Dev. Biol. 364, 32-41 (2012).

69. Bejsovec, A. \& Wieschaus, E. Signaling activities of the Drosophila wingless gene are separately mutable and appear to be transduced at the cell surface. Genetics 139, 309-320 (1995).

70. Menck, K. et al. Induction and transport of Wnt 5a during macrophageinduced malignant invasion is mediated by two types of extracellular vesicles. Oncotarget 4, 2057-2066 (2013).

71. Luga, V. et al. Exosomes mediate stromal mobilization of autocrine Wnt-PCP signaling in breast cancer cell migration. Cell 151, 1542-1556 (2012).

72. Panakova, D., Sprong, H., Marois, E., Thiele, C. \& Eaton, S. Lipoprotein particles are required for Hedgehog and Wingless signalling. Nature 435, 58-65 (2005)

73. Pfeiffer, S., Ricardo, S., Manneville, J. B., Alexandre, C. \& Vincent, J. P. Producing cells retain and recycle Wingless in Drosophila embryos. Curr. Biol. 12, 957-962 (2002).

74. Pulipparacharuvil, S. et al. Drosophila Vps16A is required for trafficking to lysosomes and biogenesis of pigment granules. J. Cell Sci. 118, 3663-3673 (2005).

75. Giraldez, A. J., Copley, R. R. \& Cohen, S. M. HSPG modification by the secreted enzyme Notum shapes the Wingless morphogen gradient. Dev. Cell. 2, 667-676 (2002).

76. Croker, J. A., Ziegenhorn, S. L. \& Holmgren, R. A. Regulation of the Drosophila transcription factor, Cubitus interruptus, by two conserved domains. Dev. Biol. 291, 368-381 (2006)

77. Yan, R., Han, P., Miao, H., Greengard, P. \& Xu, H. The transmembrane domain of the Alzheimer's beta-secretase (BACE1) determines its late Golgi localization and access to beta -amyloid precursor protein (APP) substrate. J. Biol. Chem 276, 36788-36796 (2001).

78. Schneider, I. Cell Lines Derived from Late Embryonic Stages of DrosophilaMelanogaster. J. Embryol. Exp. Morph. 27, 353 (1972).

79. Chu, M. L. et al. structural Studies of Wnts and identification of an LRP6 binding site. Structure 21, 1235-1242 (2013). 